\title{
The former companion of hyper-velocity star S5-HVS1
}

\author{
Wenbin Lu ${ }^{\oplus}{ }^{1 \star}$ Jim Fuller ${ }^{\oplus},{ }_{1}^{1}$ Yael Raveh, ${ }^{2}$ Hagai B. Perets ${ }^{\bullet}, 2$ Ting S. Li ${ }^{\oplus}, 3+4$ Matthew W. Hosek, Jr. ${ }^{5}$ \\ and Tuan $\mathrm{Do}^{5}$ \\ ${ }^{1}$ Theoretical Astrophysics, Walter Burke Institute for Theoretical Physics, Mail Code 350-17, Caltech, Pasadena, CA 91125, USA \\ ${ }^{2}$ Physics Department, Technion - Israel Institute of Technology, Haifa 3200003, Israel \\ ${ }^{3}$ Observatories of the Carnegie Institution for Science, 813 Santa Barbara St, Pasadena, CA 91101, USA \\ ${ }^{4}$ Department of Astrophysical Sciences, Princeton University, Princeton, NJ 08544, USA \\ ${ }^{5}$ UCLA Galactic Center Group, Physics and Astronomy Department, University of California, Los Angeles, CA 90024, USA
}

Accepted 2021 February 11. Received 2021 January 18; in original form 2020 May 24

\begin{abstract}
The hyper-velocity star S5-HVS1, ejected $5 \mathrm{Myr}$ ago from the Galactic Centre at $1800 \mathrm{~km} \mathrm{~s}^{-1}$, was most likely produced by tidal break-up of a tight binary by the supermassive black hole SgrA $*$. Taking a Monte Carlo approach, we show that the former companion of S5-HVS1 was likely a main-sequence star between 1.2 and $6 \mathrm{M}_{\odot}$ and was captured into a highly eccentric orbit with pericentre distance in the range of 1-10 au and semimajor axis about $10^{3}$ au. We then explore the fate of the captured star. We find that the heat deposited by tidally excited stellar oscillation modes leads to runaway disruption if the pericentre distance is smaller than about 3 au. Over the past $5 \mathrm{Myr}$, its angular momentum has been significantly modified by orbital relaxation, which may stochastically drive the pericentre inwards below 3 au and cause tidal disruption. We find an overall survival probability in the range 5 per cent to 50 per cent, depending on the local relaxation time in the close environment of the captured star, and the initial pericentre at capture. The pericentre distance of the surviving star has migrated to 10-100 au, making it potentially the most extreme member of the S-star cluster. From the ejection rate of S5-HVS1-like stars, we estimate that there may currently be a few stars in such highly eccentric orbits. They should be detectable (typically $K_{\mathrm{s}} \lesssim 18.5 \mathrm{mag}$ ) by the GRAVITY instrument and by future Extremely Large Telescopes and hence provide an extraordinary probe of the spin of SgrA*.
\end{abstract}

Key words: black hole physics - stars: kinematics and dynamics - stars: oscillations - Galaxy: centre.

\section{INTRODUCTION}

Koposov et al. (2020) reported a hyper-velocity star (HVS) S5-HVS1 that is confidently associated with the Galactic Centre (GC). The HVS is an A-type main-sequence star of about $2.3 \mathrm{M}_{\odot}$ and its inferred ejection speed from the GC is $v_{\mathrm{e}} \simeq 1800 \mathrm{~km} \mathrm{~s}^{-1}$. The ejection speed being much larger than the surface escape speed of the star rules out the ejection scenarios of supernova explosion in a close binary or dynamical encounters between binaries (see e.g. Perets \& Šubr 2012). The most likely mechanism is the tidal break-up of a tight binary system by the supermassive black hole (BH) SgrA* (Hills 1988), where one star is ejected to infinity at high speed and the other is bound. The ejection speed is roughly $v_{\mathrm{e}} \sim v_{\text {orb }}\left(M / m_{t}\right)^{1 / 6}(\mathrm{Yu}$ $\&$ Tremaine 2003), where $v_{\text {orb }}$ is the internal orbital speed of the binary and $M / m_{\mathrm{t}}$ is the $\mathrm{BH}$-to-binary mass ratio, so a supermassive $\mathrm{BH}$ is required for the observed ejection speed. Assuming the GC origin, the current location and speed of S5-HVS1 give a traveltime of about $5 \mathrm{Myr}$ since ejection (Koposov et al. 2020).

It has been proposed (e.g. Gould \& Quillen 2003; Perets, Hopman \& Alexander 2007) that the above Hills mechanism is responsible for the population of both HVSs (Brown et al. 2005) and the young stars ${ }^{1}$ in the inner $0.04 \mathrm{pc}$ (the 'S-star cluster') in roughly isotropic distribution with high eccentricities (Genzel et al. 2010; Gillessen et al. 2017). If we make a simple assumption that S5HVS1 was initially in an equal-mass binary, then the captured star has a specific binding energy of $-v_{\mathrm{e}}^{2} / 2$, which corresponds to an orbit with semimajor axis (SMA) of about $1000 \mathrm{au}$ - similar to the S2 star. Since the eccentricity of the captured star is roughly $1-e$ $\lesssim\left(m_{\mathrm{t}} / M\right)^{1 / 3} \sim 0.01$, the pericentre distance is of the order of $10 \mathrm{au}$ or less - a factor of 10 smaller than that of the S2 star (GRAVITY Collaboration 2020). We see that the captured star should be (at least temporarily) an extreme member of the S-star cluster. Its relativistic orbit provides an extraordinary probe of the spin of SgrA* (Waisberg et al. 2018), provided that it survives until today.

However, the captured star may have been tidally disrupted in the past $5 \mathrm{Myr}$ as a result of the following two processes. First, for each pericentre passage, internal stellar oscillation modes are excited by the tidal potential (Press \& Teukolsky 1977), and the mode energy may be dissipated into heat as it grows past an instability threshold (Kumar \& Goodman 1996; Wu \& Goldreich 2001; Weinberg et al. 2012). This cumulative tidal heating may lead

\footnotetext{
${ }^{1}$ See Alexander (2005) and Genzel, Eisenhauer \& Gillessen (2010) for a discussion of the many proposed formation scenarios for these stars, and Perets (2009) and Perets \& Gualandris (2010) for constraints on such origins.
}

${ }^{\star}$ E-mail: wenbinlu@ caltech.edu
†NHFP Einstein Fellow. 
to runaway radius expansion and eventual tidal disruption of the star (Alexander \& Morris 2003; Antonini, Lombardi \& Merritt 2011; Li \& Loeb 2013). Additionally, in the $N$-body environment near $\operatorname{SgrA} *$, the star experiences numerous scattering by other objects (most importantly stellar-mass BHs) in the process of relaxation and the resulting angular momentum diffusion may push the pericentre inwards and cause tidal disruption (Perets et al. 2007, 2009).

In this paper, we first provide general constraints on the mass and orbital parameters of the captured star by studying the binary break-up process in Section 2. Then in Section 3, we take a statistical approach to obtain the probability distribution of the mass and orbital parameters of the captured star. Taking the results from Section 3 as initial conditions (ICs), we model the interior and orbital evolution of the star for $5 \mathrm{Myr}$ to study its survival likelihood, taking into account tidal heating (Section 4.1) and orbital relaxation (Section 4.2). We discuss the implications and the prospects of future observation in Section 5. A summary is provided in Section 6. We use the convention $Q=10^{x} Q_{x}$ in CGS units, except that all masses are denoted in units of solar mass $\mathrm{M}_{\odot}$. We take the mass of SgrA* and its distance to be $M=4.26 \times 10^{6} \mathrm{M}_{\odot}$ and $D=8.25 \mathrm{kpc}$ (GRAVITY Collaboration 2020).

\section{BINARY BREAK-UP}

We consider a binary system of component masses $m_{\mathrm{e}}$ (star 1 , the ejected one) and $m_{\mathrm{b}}$ (star 2 , the bound one), with an SMA $a$, initially in a parabolic orbit near the $\mathrm{BH}$ with a pericentre distance $r_{\mathrm{p}}$. More realistically, the initial orbit is hyperbolic with an asymptotic (thermal) speed of a few hundred $\mathrm{km} \mathrm{s}^{-1}$. The initial kinetic energy is much smaller than the energy of the ejected $s t a r m_{\mathrm{e}} v_{\mathrm{e}}^{2} / 2$ and is hence negligible for our purpose. For the same reason, we also ignore the binding energy of the initial binary. The original binary orbit is assumed to be circular, because, as we will show later, their initial separation is constrained to be about a few times the size of ejected star and hence their orbits should have been tidally circularized.

Our coordinate system is centred on the $\mathrm{BH}$, which stays at rest. The binary centre of mass position is denoted as $\mathbf{r}$, and each star's position with respect to the centre of mass is $\mathbf{a}_{1}$ and $\mathbf{a}_{2}$, as shown in Fig. 1. The separation between the two stars is $a=a_{1}+a_{2}$. We define a unit vector $\mathbf{e}_{21}$ pointing from star 2 to star 1 , and then the stellar position vectors can be written as

$\mathbf{a}_{1}=\frac{m_{\mathrm{b}} a}{m_{\mathrm{e}}+m_{\mathrm{b}}} \mathbf{e}_{21}, \mathbf{a}_{2}=-\frac{m_{\mathrm{e}} a}{m_{\mathrm{e}}+m_{\mathrm{b}}} \mathbf{e}_{21}$.

The BH's tidal acceleration on star 1 is

$\mathbf{g}_{\mathrm{T}, 1}=\mathbf{g}\left(\mathbf{r}+\mathbf{a}_{1}\right)-\mathbf{g}(\mathbf{r})=\frac{G M a_{1}}{r^{3}}\left(3 \cos \theta \hat{\mathbf{r}}-\mathbf{e}_{21}\right)$,

where we have ignored higher order terms and defined

$\cos \theta \equiv \hat{\mathbf{r}} \cdot \mathbf{e}_{21}$.

This should be compared with the gravitational attraction from star 2 on $\operatorname{star} 1$

$\mathbf{g}_{1}=-\frac{G m_{\mathrm{b}}}{a^{2}} \mathbf{e}_{21}=-\frac{G\left(m_{\mathrm{e}}+m_{\mathrm{b}}\right) a_{1}}{a^{3}} \mathbf{e}_{21}$.

We consider the binary to be instantaneously disrupted when $g_{1}=$ $g_{\mathrm{T}, 1}$ (equivalent to $g_{2}=g_{\mathrm{T}, 2}$ ), and this defines the binary tidal breakup radius

$r_{\mathrm{T}}=f a\left(\frac{M}{m_{\mathrm{e}}+m_{\mathrm{b}}}\right)^{1 / 3}$,

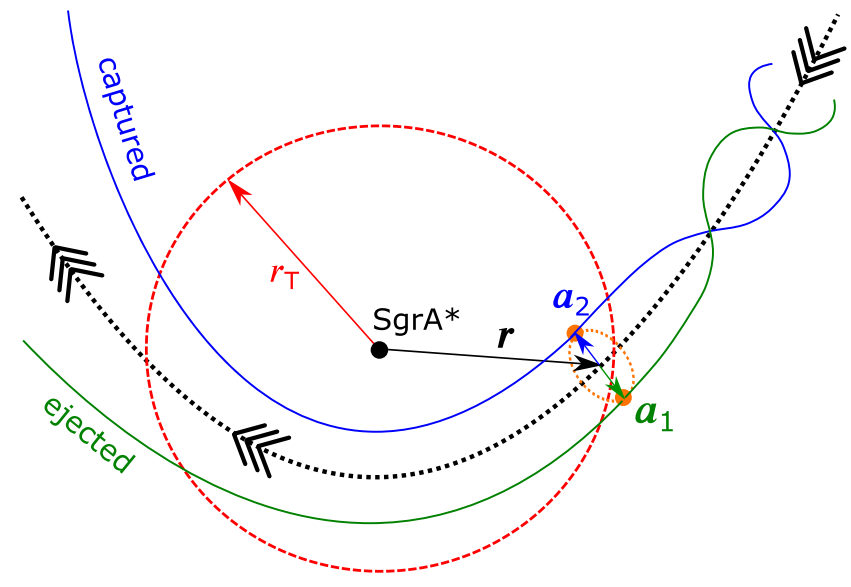

Figure 1. Sketch of the binary break-up process (not to scale). The binary centre of mass is initially in a nearly parabolic orbit (black dotted line with arrows). When the system reaches below the binary tidal break-up radius $r_{\mathrm{T}}$ (red dashed circle), tidal forces from SgrA* tear the two stars apart, and the one closer to the $\mathrm{BH}$ (blue trajectory) is captured and the farther one is ejected at high speed (green trajectory). The vector from the BH to the binary centre of mass is denoted as $\mathbf{r}$, and the vectors from the binary centre of mass to each star are $\mathbf{a}_{1}$ and $\mathbf{a}_{2}$.

where $f$ is a numerical factor of order unity given by

$f=\left|3 \cos \theta \hat{\mathbf{r}}-\mathbf{e}_{21}\right|=\left(1+3 \cos ^{2} \theta\right)^{1 / 2} \in[1,2]$.

The impulse approximation is reasonable since the tidal acceleration is a very strong function of distance to the $\mathrm{BH}$ such that $g_{1} \gg g_{\mathrm{T}, 1}$ at distance $r \gtrsim 1.3 r_{\mathrm{T}}$ and $g_{1} \ll g_{\mathrm{T}, 1}$ at $r \lesssim 1.3 r_{\mathrm{T}}$.

At the binary break-up radius, both stars have nearly the same speed $v_{\mathrm{T}}=\sqrt{2 G M / r_{\mathrm{T}}}$ or specific kinetic energy $G M / r_{\mathrm{T}}$. Their potential energies are different due to different positions, with star 2's potential energy being $-G M / r_{\mathrm{T}}\left(1+a_{2} \cos \theta / r_{\mathrm{T}}\right)$ and star 1 's being $-G M / r_{\mathrm{T}}\left(1-a_{1} \cos \theta / r_{\mathrm{T}}\right)$. Our assumption of impulsive disruption means each one's total orbital energy is conserved right after the binary break-up, so we can write the two orbital energies as

$\mathcal{E}_{1}=m_{\mathrm{e}} v_{\mathrm{e}}^{2} / 2$,

$\frac{\mathcal{E}_{2}}{m_{\mathrm{b}}}=\frac{G M}{r_{\mathrm{T}}}-\frac{G M}{r_{\mathrm{T}}}\left(1+\frac{a_{2} \cos \theta}{r_{\mathrm{T}}}\right)=-\frac{G M}{r_{\mathrm{T}}^{2}} \frac{m_{\mathrm{e}} a}{m_{\mathrm{e}}+m_{\mathrm{b}}} \cos \theta$,

and total energy conservation gives

$\mathcal{E}_{1}+\mathcal{E}_{2}=0$.

Combining equations (5), (7), (8), and (9), we obtain

$a=\frac{2 \sqrt{3} \cos \theta}{f^{2}} a_{\max }, \quad r_{\mathrm{T}}=\frac{2 \cos \theta}{f} r_{\mathrm{T}, \max }$,

where, for convenience, we have defined the maximum allowed binary separation $a$ as when $\cos \theta=1 / \sqrt{3}$ (and $f=\sqrt{2}$ ),

$$
\begin{aligned}
a_{\max } & =\left(\frac{M}{m_{\mathrm{e}}+m_{\mathrm{b}}}\right)^{1 / 3} \frac{G m_{\mathrm{b}}}{v_{\mathrm{e}}^{2}} \\
& =3.7 \times 10^{11} \mathrm{~cm} \frac{m_{\mathrm{b}} M_{6.6}^{1 / 3}}{\left(m_{\mathrm{b}}+m_{\mathrm{e}}\right)^{1 / 3}} v_{\mathrm{e}, 1800}^{-2}
\end{aligned}
$$


and the maximum allowed binary break-up radius $r_{\mathrm{T}}$ as when $\cos \theta$ $=1($ and $f=2)$,

$$
\begin{aligned}
r_{\mathrm{T}, \max } & =\left(\frac{M}{m_{\mathrm{e}}+m_{\mathrm{b}}}\right)^{2 / 3} \frac{G m_{\mathrm{b}}}{v_{\mathrm{e}}^{2}} \\
& =1.0 \times 10^{14} \mathrm{~cm} \frac{m_{\mathrm{b}} M_{6.6}^{2 / 3}}{\left(m_{\mathrm{e}}+m_{\mathrm{b}}\right)^{2 / 3}} v_{\mathrm{k}, 1800}^{-2} .
\end{aligned}
$$

The binary break-up radius should be compared to the tidal disruption radius of star 1 ,

$r_{\mathrm{T}, \mathrm{e}} \equiv R_{\mathrm{e}}\left(\frac{M}{m_{\mathrm{e}}}\right)^{1 / 3} \simeq\left(1.3 \times 10^{13} \mathrm{~cm}\right) m_{\mathrm{e}}^{1 / 3} M_{6.6}^{1 / 3}$,

where we have adopted a simple mass-radius relation ${ }^{2}$ of $R \simeq 1.2$ $\mathbf{R}_{\odot}\left(m / \mathbf{M}_{\odot}\right)^{2 / 3}$, appropriate for massive $\left(\gtrsim 1.5 \mathrm{M}_{\odot}\right)$ main-sequence stars at the inferred age $(\sim 50 \mathrm{Myr})$ and metallicity $([\mathrm{Fe} / \mathrm{H}] \sim$ 0.3) of S5-HVS1 (Koposov et al. 2020). The highly centrally concentrated density profile of massive $\left(\gtrsim 1.5 \mathrm{M}_{\odot}\right)$ stars requires deeper penetration down to $0.5 r_{\mathrm{T}, \mathrm{e}}$ to cause major disruption where the star loses $\gtrsim 50$ per cent of its mass (Guillochon \& Ramirez-Ruiz 2013; Mainetti et al. 2017; Ryu et al. 2020). Since the ejected star survived the binary break-up, we require the pericentre distance of the initial binary to be more than $0.5 r_{\mathrm{T}}$, e i.e.

$r_{\mathrm{p}}>0.5 r_{\mathrm{T}, \mathrm{e}}$.

We note that the bound star may have been disrupted, and the probability will be quantified in the next section. We also require that the two stars are separated by

$a>2 \max \left(R_{\mathrm{e}}, R_{\mathrm{b}}\right)$,

so that the radius of the more massive star (with larger radius and lower density) is smaller than the effective radius of its Roche lobe (Eggleton 1983). The precise lower limit for the binary separation depends on the deformation of the stars near Roche lobe filling. At even shorter binary separation, the more massive star will fill up its Roche lobe and the system is unstable (the detailed consequence is diverse, e.g. Vanbeveren et al. 1998).

We can rule out very low mass $m_{\mathrm{b}}$ by requiring $0.5 r_{\mathrm{T}, \mathrm{e}}<r_{\mathrm{T}, \max }$ and $2 \max \left(R_{\mathrm{e}}, R_{\mathrm{b}}\right)<a_{\max }$, as shown in Fig. 2 . We find that $m_{\mathrm{b}}>1.2 \mathrm{M}_{\odot}$ is generally required. It is possible that the bound star is a massive white dwarf (as the remnant of a massive star $\sim 8 \mathrm{M}_{\odot}$, see Cummings et al. 2018), but this requires an additional common-envelope evolution so as to bring the orbital separation down to a few solar radii before the binary break-up. A neutron star or BH is also possible, but the probability is even smaller, given the steep Salpeter/Kroupa mass function of the progenitor star and that the binary system may be destroyed by the natal kick and mass-loss during the supernova. For the above reasons, we only consider the bound star being a main-sequence star.

The SMA of the bound star after binary break-up is denoted as $a_{\mathrm{b}}$ and is given by $\mathcal{E}_{2} / m_{\mathrm{b}}=-G M / 2 a_{\mathrm{b}}$, i.e.

$a_{\mathrm{b}}=\frac{G M m_{\mathrm{b}}}{m_{\mathrm{e}} v_{\mathrm{e}}^{2}}=1.1 \times 10^{3} \mathrm{au} \frac{m_{\mathrm{b}} M_{6.6}}{m_{\mathrm{e}}} v_{\mathrm{e}, 1800}^{-2}$

\footnotetext{
${ }^{2}$ Our results are only weakly affected by this approximate relation, because it is only used to set the boundaries of the minimum mass of the captured star and the pericentre distance of the pre-break-up orbit. Disruptions due to cumulative tidal heating are treated more accurately based on numerical modelling of the stellar interior structure (see Section 4.1).
}

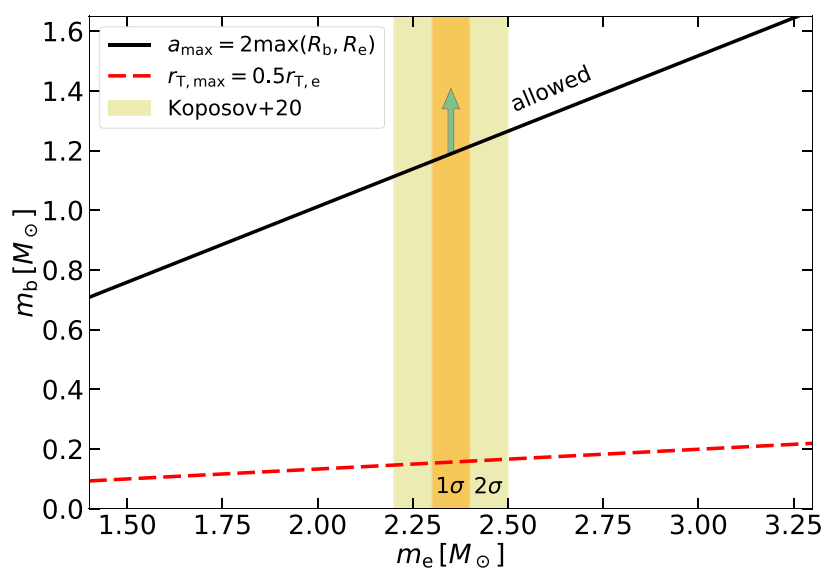

Figure 2. The minimum mass of the bound star as constrained by $0.5 r_{\mathrm{T}} \mathrm{e}$ $<r_{\mathrm{T}, \max }$ (the ejected star is not tidally disrupted) and $a_{\max }>2 \max \left(R_{\mathrm{e}}, R_{\mathrm{b}}\right)$ (the two stars must be separated before the break-up). The latter is a stronger constraint, so the bound star's mass $m_{\mathrm{b}}$ must be above the solid black line. Shown in yellow is the spectroscopic+photometric measurements of $m_{\mathrm{e}}$, with $1 \sigma$ and $2 \sigma$ errors.

which is similar to that of the S2 star (Gillessen et al. 2017; Do et al. 2019). The Keplerian orbital period is

$P=18 \mathrm{yr}\left(\frac{m_{\mathrm{b}}}{m_{\mathrm{e}}}\right)^{3 / 2} M_{6.6} v_{\mathrm{e}, 1800}^{-3}$.

However, the bound star's pericentre is much closer to the BH than the S2 star (whose $r_{\mathrm{p}} \simeq 120 \mathrm{au}$ ). From the binary break-up criterion $r_{\mathrm{p}} \leq r_{\mathrm{T}}$, we know that the orbit of the bound star is highly eccentric $1-e=r_{\mathrm{p}} / a \sim 10^{-3}$ to $10^{-2}$.

\section{MONTE CARLO MODELLING}

In this section, we statistically constrain the pericentre and mass of the captured star. Previous works (e.g. Bromley et al. 2006; Zhang, Lu $\&$ Yu 2013) rely on a large number of 3-body scattering experiments for ICs drawn from (assumed) distributions of binary masses, SMAs of the inner binary orbit, and angular momentum of the BH-binary orbit. Our approach is different and simpler in that we make use of all the known information about the ejected star S5-HVS1 instead of drawing random ICs blindly.

In the case where the ejected star's kinetic energy is much greater than the initial binding energy of the binary, each member may be ejected at equal probability (Sari, Kobayashi \& Rossi 2010). The moment the binary reaches the binary break-up radius, whichever star farther away from the $\mathrm{BH}$ is ejected and the closer one is captured, depending on random orbital phase. The inner binary orbit is randomly oriented with respect to the orbit around the $\mathrm{BH}$ and the orientation is independent of $m_{\mathrm{b}}$ and the pericentre distance $r_{\mathrm{p}}$. We denote the probability density function (PDF) of a quantity $x$ as $\mathcal{P}(x)$ and the corresponding cumulative density function (CDF) is $\mathcal{F}(x)=\int^{x} \mathcal{P}\left(x^{\prime}\right) \mathrm{d} x^{\prime}$. Thus, the joint probability of all three parameters is given by

$\mathcal{P}\left(m_{\mathrm{b}}, r_{\mathrm{p}}, \theta\right)=\mathcal{P}\left(m_{\mathrm{b}}\right) \times \mathcal{P}\left(r_{\mathrm{p}}\right) \times \mathcal{P}(\theta) \times \mathcal{C}\left(m_{\mathrm{b}}, r_{\mathrm{p}}, \theta\right)$,

where $\mathcal{P}\left(m_{\mathrm{b}}\right)$ describes the PDF of $m_{\mathrm{b}}$ for a known $m_{\mathrm{e}}$ (to be quantified below), $\mathcal{P}\left(r_{\mathrm{p}}\right)$ describes the PDF of the pericentre distance of the initial binary orbit near SgrA* (which in turn depends on the processes scattering the binaries into the $\mathrm{SgrA} *$, e.g. loss-cone dynamics discussed later on), $\mathcal{P}(\theta)$ is the PDF of $\theta \equiv \hat{\mathbf{r}} \cdot \mathbf{e}_{21}$ and is 
considered to be isotropically distributed

$\mathcal{P}(\theta) \propto \sin \theta$,

and finally $\mathcal{C}\left(m_{\mathrm{b}}, r_{\mathrm{p}}, \theta\right)$ contains the various constraints from known information as summarized below:

(i) The bound star's mass is constrained to be $m_{\mathrm{b}}<m_{\mathrm{b}, \max }=6$ $\mathrm{M}_{\odot}$ by the age of the binary system before break-up (taken to be $50 \mathrm{Myr})$.

(ii) The binary break-up radius $r_{\mathrm{T}}$ (and the initial binary separation a) is given by equation (10) as required by the measured ejection speed $v_{\mathrm{e}}$.

(iii) The pericentre radius $r_{\mathrm{p}} \leq r_{\mathrm{T}}$ such that binary break-up occurs.

(iv) Star 1 is ejected and hence $\theta<\pi / 2$.

(v) The pericentre radius $r_{\mathrm{p}}>0.5 r_{\mathrm{T}, \mathrm{e}}$ such that the ejected star is not tidally disrupted.

(vi) The initial binary is separated by $a>2 \max \left(R_{\mathrm{e}}, R_{\mathrm{b}}\right)$.

We note that the order of drawing $m_{\mathrm{b}}, r_{\mathrm{p}}$, and $\theta$ is unimportant, since they are independent (except for the constraints above). We reject the entire Monte Carlo (MC) sample of $\left(m_{\mathrm{b}}, r_{\mathrm{p}}, \theta\right)$ if any of these constraints is violated and start over. In the following, we describe our choices of $\mathcal{P}\left(m_{\mathrm{b}}\right)$ and $\mathcal{P}\left(r_{\mathrm{p}}\right)$.

We first randomly draw the mass of the bound star $m_{\mathrm{b}}$ according to a power-law binary mass ratio distribution $\mathcal{P}(q) \propto q^{\alpha}$ for each particular primary mass, and our fiducial power-law index is $\alpha=0$. This is motivated by statistical studies of short period binaries with mass ratio between 0.3 and 1 by Moe \& Di Stefano (2017). We find that our results are only weakly affected for other choices of $\alpha=$ -0.5 and 0.5 , because only a small range of mass ratio $0.4 \lesssim q<$ 1 is allowed by the physical constraints of the system. For the same reason, our results are insensitive to possible Case A mass transfer before the binary break-up, which changes the mass ratio by mass transfer between the two stars while both are on the main sequence.

If the ejected star is the primary $\left(m_{\mathrm{b}}<m_{\mathrm{e}}\right.$, which occurs half of the time), then we have

$\mathcal{P}\left(m_{\mathrm{b}}\right)=\frac{1+\alpha}{2 m_{\mathrm{e}}}\left(m_{\mathrm{b}} / m_{\mathrm{e}}\right)^{\alpha}$ for $m_{\mathrm{b}}<m_{\mathrm{e}}$.

If the bound star is the primary $\left(m_{\mathrm{b}}>m_{\mathrm{e}}\right)$, then we first consider the mass distribution of the primary to be a power-law $\propto m_{\mathrm{b}}^{-2.3}$ with a Salpeter/Kroupa initial mass function (IMF) slope and then multiply by the probability of mass ratio $q=m_{\mathrm{e}} / m_{\mathrm{b}}$, i.e.

$\mathcal{P}\left(m_{\mathrm{b}}\right)=0.5(2.3+\alpha)\left(m_{\mathrm{b}} / m_{\mathrm{e}}\right)^{-2.3-(1+\alpha)}$ for $m_{\mathrm{b}}>m_{\mathrm{e}}$.

The CDF combining the two cases is

$\mathcal{F}\left(m_{\mathrm{b}}\right)= \begin{cases}0.5\left(m_{\mathrm{b}} / m_{\mathrm{e}}\right)^{1+\alpha} & \text { for } m_{\mathrm{b}}<m_{\mathrm{e}}, \\ 1-0.5\left(m_{\mathrm{b}} / m_{\mathrm{e}}\right)^{-(2.3+\alpha)} & \text { for } m_{\mathrm{b}}>m_{\mathrm{e}} .\end{cases}$

The random draw of $m_{\mathrm{b}}$ from above is immediately rejected if $r_{\mathrm{T}, \max }$ $<0.5 r_{\mathrm{T}, \mathrm{e}}$ or $a_{\mathrm{b}, \max }<2 \max \left(R_{\mathrm{e}}, R_{\mathrm{b}}\right)$.

The maximum mass of the bound star $m_{\mathrm{b}, \text { max }}$ has large uncertainty because the age of the binary system is only weakly constrained to be between 30 and $100 \mathrm{Myr}$ (at $1 \sigma$; Koposov et al. 2020). We have tested different choices of $m_{\mathrm{b} \text {, max }}$ between 5 and $8 \mathrm{M}_{\odot}$ (corresponding to different binary ages) and found the results to be qualitatively similar. A star between $m_{\mathrm{b}, \text { max }}$ and $\sim 8 \mathrm{M}_{\odot}$ would have evolved off the main sequence to become a white dwarf before the binary break-up. Using the initial-final mass relationship from Cummings et al. (2018), we find that the probability of the bound star being a white dwarf with mass above constraint from the minimum binary separation (Fig. 2) is about 1 per cent in the full loss-cone case and even smaller in the empty loss-cone case. In reality, this probability should be further reduced, because a common-envelope evolution is require to shrink the binary separation down to a few solar radii. A star $\gtrsim 8 \mathrm{M}_{\odot}$ should have exploded and the binary system may have been destroyed by the mass-loss and natal kick during the supernova. We ignore these cases, making a small error given the steepness of the mass function slope. Nevertheless, if the captured star is a compact object, this channel contributes a fraction of extreme mass ratio inspirals (EMRIs).

We also note that the true mass ratio distribution and primary mass function are highly uncertain for tight binaries near the GC, but our approach is general and can be improved when more information is available. For instance, if S5-HVS1 was formed under a top-heavy IMF slope of -1.7 as suggested by the population of young massive stars from $\mathrm{SgrA} *$ (Lu et al. 2013), then the probability of the captured star being a neutron star or BH may be as large as 5 per cent. However, our results are only weakly affected if the captured companion of S5-HVS1 is a main-sequence star, the case that we focus on.

The pericentre distance distribution $\mathcal{P}\left(r_{\mathrm{p}}\right)$ is more complicated. Typically, binary systems or single stars fall towards the centre from large distances near the BH's sphere of influence $r_{\mathrm{h}}=G M / \sigma^{2}$ where the typical angular momentum is $J_{\mathrm{c}} \simeq \sigma r_{\mathrm{h}}$ (for near circular orbits), and $\sigma$ is the velocity dispersion at $r_{\mathrm{h}}$. The specific angular momentum of the loss-cone orbit is

$J_{\mathrm{lc}}=\sqrt{2 G M r_{\mathrm{T}}}$,

and, for isotropic velocity distribution, only a small fraction $\left(J_{\mathrm{lc}} / J_{\mathrm{c}}\right)^{2}$ $\simeq 2 r_{\mathrm{T}} / r_{\mathrm{h}}$ of binary systems have sufficiently high eccentricity as to reach the binary break-up radius $r_{\mathrm{T}}$. These systems are destroyed in one orbital time $P\left(r_{\mathrm{h}}\right) \simeq 2 \pi \sqrt{r_{\mathrm{h}}^{3} / G M}$, during which other systems may be scattered into the loss cone to maintain a quasi-steady breakup rate. A typical system's angular momentum and orbital energy change by order unity in a relaxation time (Binney \& Tremaine 1987; Merritt et al. 2011)

$t_{\mathrm{rel}}\left(r_{\mathrm{h}}\right) \simeq 0.2 \frac{M^{2}}{\left\langle m^{2}\right\rangle} \frac{P\left(r_{\mathrm{h}}\right)}{N\left(r_{\mathrm{h}}\right) \ln \Lambda}$,

where $N\left(r_{\mathrm{h}}\right) \simeq 2 M /\langle m\rangle$ is the number of stars near $r_{\mathrm{h}},\langle m\rangle$ and $\left\langle m^{2}\right\rangle$ are the first and second moments of the stellar mass spectrum, and $\ln \Lambda \sim 10$ is the Coulomb logarithm. The amount of angular momentum diffusion due to 2-body encounters during $P\left(r_{\mathrm{h}}\right)$ is $\Delta J^{2} / J_{\mathrm{c}}^{2} \simeq P\left(r_{\mathrm{h}}\right) / t_{\mathrm{rel}}$.

There are two dynamical regimes of loss-cone filling, depending on the ratio between the mean change in angular momentum per orbit $\Delta J$ and $J_{\text {lc }}$ (Lightman \& Shapiro 1977). If $\Delta J \ll J_{\text {lc }}$, then the loss cone is largely empty and binary break-up mainly occurs at the boundary for systems with $J \simeq J_{\mathrm{lc}}$. The binary break-up rate is given by the rate at which systems diffuse into the loss cone $\sim N\left(r_{\mathrm{h}}\right) / t_{\text {rel }}$. On the other hand, if $\Delta J \gg J_{1 \mathrm{c}}$, the loss cone is fully populated and binary break-up occurs for any angular momentum $J \leq J_{\text {lc }}$ (deeply penetrating orbits with $r_{\mathrm{p}} \ll r_{\mathrm{T}}$ are allowed). In this case, the binary break-up rate is $\sim N\left(r_{\mathrm{h}}\right)\left(J_{\mathrm{lc}} / J_{\mathrm{c}}\right)^{2} / P\left(r_{\mathrm{h}}\right) \simeq N\left(r_{\mathrm{h}}\right) r_{\mathrm{T}} / P\left(r_{\mathrm{h}}\right) r_{\mathrm{h}}$. For our GC, the loss cone filling depends on the critical ratio

$\frac{\Delta J^{2}}{J_{\mathrm{lc}}^{2}} \simeq \frac{5 \ln \Lambda}{2} \frac{r_{\mathrm{h}}}{r_{\mathrm{T}}} \frac{\left\langle m^{2}\right\rangle}{M\langle m\rangle}$.

Taking $r_{\mathrm{h}} \simeq 2 \mathrm{pc}, r_{\mathrm{T}} \simeq 5 \mathrm{au}$ (for tight binaries only), the mean stellar mass $\langle m\rangle \simeq 0.5 \mathrm{M}_{\odot}$, the mean squared stellar mass $\left\langle m^{2}\right\rangle \simeq 1 \mathrm{M}_{\odot}^{2}$, we find that the loss cone should be full with $\Delta J^{2} / J_{\text {lc }}^{2} \sim 10$ near $r_{\mathrm{h}}$ for tight binaries considered in this work. Other processes such as resonant relaxation and non-spherical/time-dependent gravitational potential, may modify the angular momentum diffusion rate 


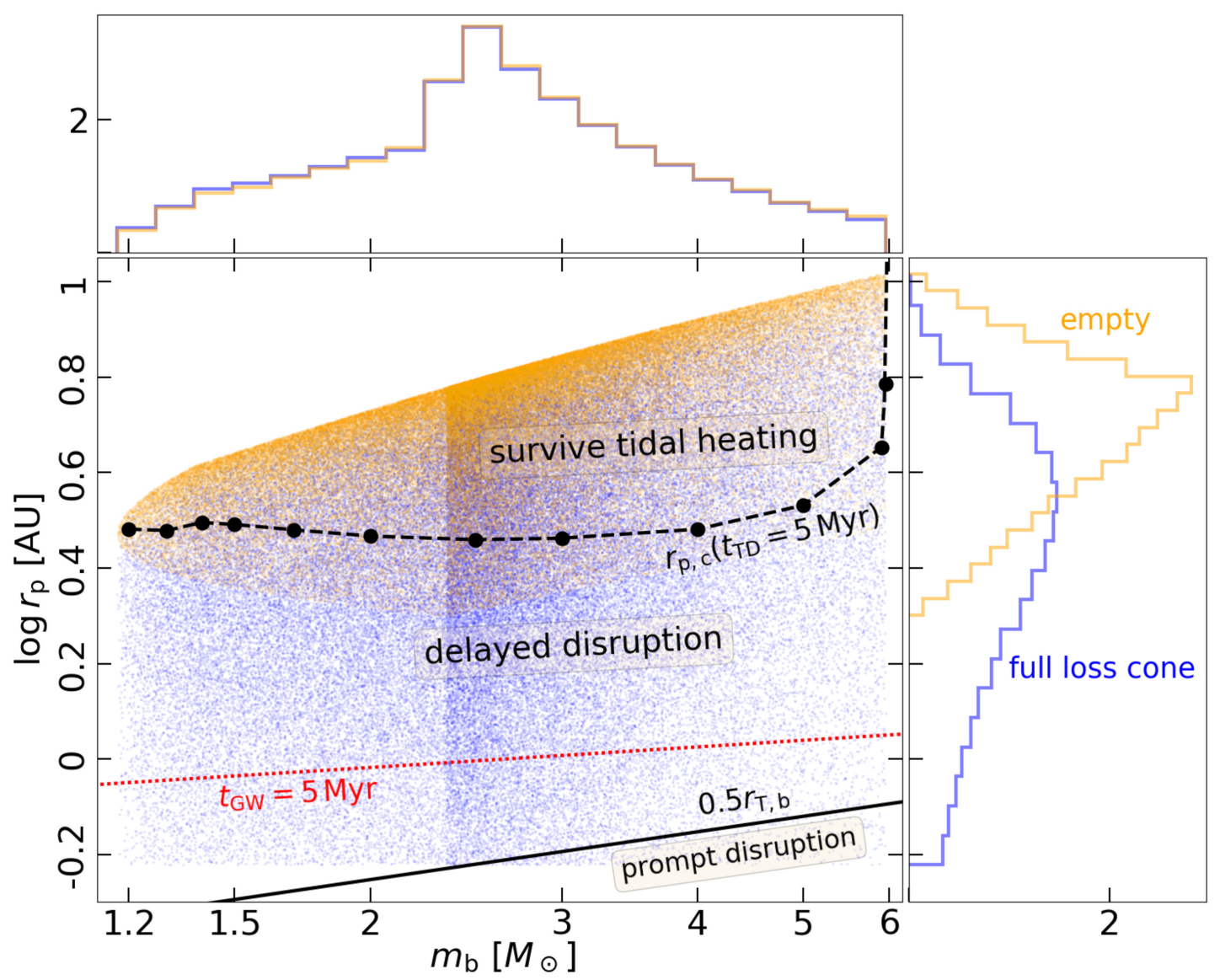

Figure 3. MC samples $\left(N=10^{5}\right)$ of the mass $m_{\mathrm{b}}$ and pericentre $r_{\mathrm{p}}$ of the bound star. We take the ejected star's mass and ejection velocity to be $m_{\mathrm{e}}=2.35 \mathrm{M} \odot$ and $v_{\mathrm{e}}=1800 \mathrm{~km} \mathrm{~s}^{-1}$, BH mass $M=4.26 \times 10^{6} \mathrm{M}_{\odot}$, and the binary mass-ratio distribution slope $\alpha=0$. The orange and blue samples correspond to empty and full loss cones, respectively. The cutoff towards the top, bottom, left and right sides of the $m_{\mathrm{b}}-r_{\mathrm{p}}$ parameter space are due to constraints by the ejection velocity $v_{\mathrm{e}}$, the ejected star's survival of tidal disruption, initial binary being separated at $a>2 \max \left(R_{\mathrm{e}}, R_{\mathrm{b}}\right)$, and main-sequence age of the ejected star, respectively. The black solid line denotes $0.5 r_{\mathrm{T}}$, , below which the bound star is promptly disrupted during the binary break-up. The filled black circles denote the critical pericentre distance $r_{\mathrm{p}, \mathrm{c}}$ below which the star is tidally disrupted within $5 \mathrm{Myr}$ after the binary break-up, as a result of cumulative tidal heating (see Section 4.1). The rapid increase of $r_{\mathrm{p}, \mathrm{c}}$ above $6 \mathrm{M}_{\odot}$ is because the star evolves off the main-sequence into a red giant. The exact value of main-sequence turn-over mass depends on the age of the pre-break-up binary system, which is taken to be $50 \mathrm{Myr}$ for this example. Stars above the black dashed line survive the tidal heating for at least $5 \mathrm{Myr}$, provided that their orbits are not strongly modified by scattering off other massive objects (see discussion in Section 4.2). The parameters corresponding to $\mathrm{GW}$ inspiral time $t_{\mathrm{GW}}=5 \mathrm{Myr}$ is shown by the red line.

compared to that given by 2-body relaxation (see Merritt 2013; Alexander 2017; Stone et al. 2020, for recent reviews). We note that molecular clouds, and spiral arms near the GC (a few to $10 \mathrm{pc}$ ) can enhance the rate of relaxation and hence loss-cone filling compared to stellar perturbers. The full loss-cone case is consistent with the number of young HVSs and S-stars in the GC (if the S-stars are mainly from the Hills mechanism; Perets et al. 2007; Hamers \& Perets 2017).

In the following, we draw the distribution of pericentre distance $r_{\mathrm{p}}$ by considering two extreme cases of empty and full loss cones. The reality should be somewhere in between, especially considering that binary may come from the nuclear star cluster within the central 0.5 pc from $\mathrm{SgrA} *$ (e.g. in the eccentric disc instability scenario; Madigan, Levin \& Hopman 2009; Generozov \& Madigan 2020), and in this case the loss cone has a larger size and may be closer to the empty side. We also note that even when $\Delta J \ll J_{\text {lc }}$, the phase space deeply within the loss-cone can still be populated by rare strong scatterings (Weissbein \& Sari 2017).

In the empty loss-cone case, we first draw $\theta$ from isotropic distribution. Then, the pericentre distance $r_{\mathrm{p}}$ is taken to be the largest allowed,

$r_{\mathrm{p}}=r_{\mathrm{T}}(\theta)$ for empty loss cone.

In the full loss-cone case, we first draw $r_{\mathrm{p}}$ distribution from

$\mathcal{P}\left(r_{\mathrm{p}}\right)=\left(r_{\mathrm{T}, \max }-0.5 r_{\mathrm{T}, \mathrm{e}}\right)^{-1}$ for full loss cone,

since the probability of reaching down to a distance $<r_{\mathrm{p}}$ is $\int_{0}^{r_{\mathrm{p}}} \mathcal{P}\left(r_{\mathrm{p}}\right) \mathrm{d} r_{\mathrm{p}} \propto J^{2}\left(r_{\mathrm{p}}\right) \propto r_{\mathrm{p}}$ and hence $\mathcal{P}\left(r_{\mathrm{p}}\right)$ is constant. The allowed range is $0.5 r_{\mathrm{T}, \mathrm{e}}<r_{\mathrm{p}}<r_{\mathrm{T}, \max }$ (see equations 12 and 13), so that the ejected star is not tidally disrupted and its ejection speed is $v_{\mathrm{e}}$. Then, we randomly draw $\theta$ from equation (19).

In Fig. 3, we show MC sampling of the probability distribution of $r_{\mathrm{p}}$ and $m_{\mathrm{b}}$, for two cases of empty and full loss cones. For the empty (full) loss-cone case, the median values of these two parameters are $r_{\mathrm{p}} \sim 5 \mathrm{au}(3 \mathrm{au})$ and $m_{\mathrm{b}} \sim 2.5 \mathrm{M}_{\odot}$.

The pericentre distance $r_{\mathrm{p}}$ is related to the SMA $a_{\mathrm{b}}$ by $r_{\mathrm{p}}=a_{\mathrm{b}}(1$ $-e$ ), and in extreme eccentricity limit $1-e \ll 1$, the gravitational wave (GW) inspiral time is (Peters 1964)

$t_{\mathrm{GW}}=(2.6 \mathrm{Myr}) m_{\mathrm{b}}^{-1} M_{6.6}^{-2} r_{\mathrm{p}, 13}^{7 / 2} a_{\mathrm{b}, 16}^{1 / 2}$. 
Since the SMA $a_{\mathrm{b}}$ is determined for given $m_{\mathrm{b}}$ (by equation 16), the $\mathrm{GW}$ inspiral time of the bound star only depends on the pericentre $r_{\mathrm{p}}$. There is a critical $r_{\mathrm{p}, \mathrm{GW}}$ that corresponds to an inspiral time of 5 Myr (since the binary break-up), given by

$r_{\mathrm{p}, \mathrm{GW}}=\left(1.1 \times 10^{13} \mathrm{~cm}\right)\left(\frac{t_{\mathrm{GW}}}{5 \mathrm{Myr}}\right)^{2 / 7}\left(m_{\mathrm{e}} m_{\mathrm{b}}\right)^{1 / 7} M_{6.6}^{3 / 7} v_{\mathrm{e}, 1800}^{2 / 7}$,

The orbit is strongly affected by GW only if $r_{\mathrm{p}} \lesssim r_{\mathrm{p}, \mathrm{GW}}$. We find that GW inspiral is typically unimportant for the orbital evolution of the bound star in the past 5 Myr since break-up.

\section{FATE OF THE CAPTURED STAR}

In this section, we study the fate of the bound star by grouping the MC samples into different classes in the $r_{\mathrm{p}}-m_{\mathrm{b}}$ plane as follows:

(i) If $r_{\mathrm{p}}<0.5 r_{\mathrm{T}, \mathrm{b}}$, the star is promptly disrupted.

(ii) If $0.5 r_{\mathrm{T}, \mathrm{b}}<r_{\mathrm{p}}<r_{\mathrm{p}, \mathrm{c}}$, the star experiences strong tidal heating over many orbits, expands, and is then tidally disrupted (Antonini et al. 2011; Li \& Loeb 2013). The critical radius $r_{\mathrm{p}, \mathrm{c}}$ below which cumulative tidal heating leads to expansion and disruption within $5 \mathrm{Myr}$ is calculated in Section 4.1.

(iii) If $r_{\mathrm{p}}>r_{\mathrm{p}, \mathrm{c}}$, the star is not tidally disrupted within $5 \mathrm{Myr}$, provided that its orbit is not strongly modified by scattering of other massive objects in the field. In Section 4.2, we study the angular momentum diffusion of the star in the $\mathrm{N}$-body environment near $\mathrm{SgrA} *$ and calculate the probability that the star is scattered down below $r_{\mathrm{p}, \mathrm{c}}$.

\subsection{Cumulative tidal heating}

For each pericentre passage, energy is injected into the star's normal oscillation modes via tidal excitation according to the linear perturbation theory (Press \& Teukolsky 1977)

$\Delta E=\frac{2 \pi^{2} G M^{2}}{R_{\mathrm{b}}} \sum_{l=2,3, \ldots}\left(\frac{R_{\mathrm{b}}}{r_{\mathrm{p}}}\right)^{2 l+2} \sum_{n, m}\left|Q_{n l}\right|^{2}\left|K_{n l m}\right|^{2}$,

where $(n, l, m)$ are the quantum numbers for each eigenmode ('radial' order $n$ and spherical harmonic indices $l, m$ ), the coefficient $Q_{n l}$ is a radial overlap integral for the coupling between the (assumed Newtonian) tidal potential to a given mode, and $K_{n l m}$ is a temporal overlap integral representing the coupling to the orbit. For $r_{\mathrm{p}} / r_{\mathrm{T}, \mathrm{b}} \gtrsim$ 2 , the quadrupole $(l=2)$ modes dominate, so we approximate

$\Delta \widetilde{E}=\frac{\Delta E}{G m_{\mathrm{b}}^{2} / R_{\mathrm{b}}} \approx \chi^{-6} T\left(\chi, m_{\mathrm{b}}\right), \quad \chi \equiv \frac{r_{\mathrm{p}}}{r_{\mathrm{T}, \mathrm{b}}}$,

where $\chi$ is the dimensionless pericentre distance (in units of the tidal radius $r_{\mathrm{T}, \mathrm{b}}$ ), and for highly eccentric orbits $1-e \ll 1$, the tidal coupling constant $T \equiv 2 \pi^{2} \sum_{n, m}\left|Q_{n l=2}\right|^{2}\left|K_{n l=2 m}\right|^{2}$ can be expressed as a dimensionless function of the stellar mass $m_{\mathrm{b}}$ (controlling the interior structure) and $\chi$, because nearly all the tidal interactions occur near the pericentre. For fixed stellar interior structure, $T(\chi)$ is a rapidly decreasing function for $\chi \gtrsim 1$ (Press \& Teukolsky 1977; Lee \& Ostriker 1986; McMillan, McDermott \& Taam 1987).

We follow Li \& Loeb (2013) by assuming that the mode energy is rapidly dissipated as a result of non-linear coupling to a large number of daughter modes in the radiative zone of the star (Weinberg et al. 2012). This is because gravitational scattering in the dense environment near $\operatorname{SgrA} *$ causes stochastic change in orbital period of the captured star, with a fractional change per orbit $\Delta P / P \sim \sqrt{P / t_{\text {rel }}}$, where $t_{\text {rel }}$ is the local relaxation time. For typical mode frequency $\omega \sim \sqrt{3 G M_{*} / R_{*}^{3}}$, we find $\omega \Delta P \sim$

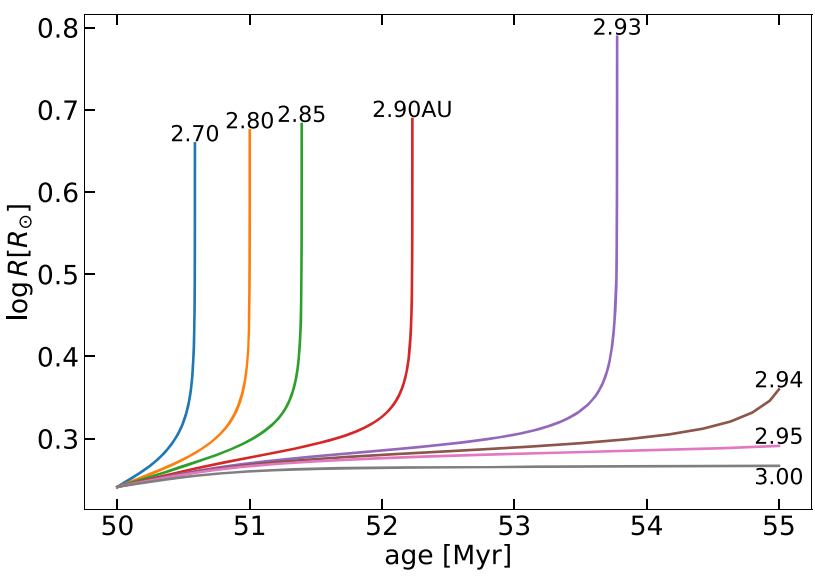

Figure 4. Radius evolution from $50 \mathrm{Myr}$ (binary break-up) to $55 \mathrm{Myr}$ (now) for the $m_{\mathrm{b}}=2 \mathrm{M}_{\odot}$ case, for different pericentre distances (in units of au) marked with each line. We take the ejected star's mass and ejection velocity to be $m_{\mathrm{e}}=2.35 \mathrm{M}_{\odot}$ and $v_{\mathrm{e}}=1800 \mathrm{~km} \mathrm{~s}^{-1}$, and the BH mass $M=$ $4.26 \times 10^{6} \mathrm{M}_{\odot}$. The orbital period is $P=14.5 \mathrm{yr}$ as given by equation (17) We find that, for $m_{\mathrm{b}}=2 \mathrm{M}_{\odot}$, the critical pericentre distance to be $r_{\mathrm{p}, \mathrm{c}} \approx 2.93$ $\mathrm{au}$, below which the star is disrupted within $5 \mathrm{Myr}$ after the tidal capture.

$30(P / 20 \mathrm{yr})^{3 / 2}\left(t_{\mathrm{rel}} / 1 \mathrm{Gyr}\right)^{-1 / 2}\left(M_{*} / 3 \mathrm{M}_{\odot}\right)^{-1} \gg 1$ for all cases considered in this work, so the system is in the chaotic tide regime where mode energy can stochastically grow (by $\Delta E_{n l m}$ on average in each orbit) and is then rapidly dissipated by non-linear effects (Mardling 1995a, b; Ivanov \& Papaloizou 2004; Vick, Lai \& Anderson 2019).

If the star reacts adiabatically and maintains hydrostatic equilibrium, then after each pericentre passage, the stellar radius increases by an amount $\Delta R_{\mathrm{b}} \sim \Delta \widetilde{E} R_{\mathrm{b}}$. Cumulative heating over a large number $\left(\sim 0.1 \Delta \widetilde{E}^{-1}\right)$ of orbits will eventually lead to the runaway expansion of the star and hence disruption. In reality, different layers of the star may radiate away the tidally generated heat, especially if the heat is deposited in the outer layers of the star where it can quickly diffuse to the surface.

We study the response of the captured star in realistic orbits using MESA (version 15140, Paxton et al. 2019) simulations. First, we calculate $T\left(\chi, m_{\mathrm{b}}\right)$ with GYRE (version 6.0.1, Townsend \& Teitler 2013) for a grid of $1.5<\chi<5$ and $1.2<m_{\mathrm{b}}<6$, where the interior structure of each star is given by $50 \mathrm{Myr}$ of MESA evolution from $Z=0.03$ metallicity zero-age main sequence (without tidal heating). Detailed procedures for calculating $T\left(\chi, m_{\mathrm{b}}\right)$ are explained in Appendix A. Metallicity only weakly modifies the tidal coupling constant for the stellar mass range of interest. Then, during the $5 \mathrm{Myr}$ of further evolution, we add an orbit-averaged tidal heating rate of $\Delta E / P$ uniformly distributed per unit mass in the radiative zone. We take into account the time dependence of $\chi$ (and hence $\Delta E$ ) due to stellar radius evolution as given by MESA. This approximation ignores the detailed structural responses of the star due to tidal heating, which only leads to a small correction as shown by Li \& Loeb (2013). In this subsection, we ignore orbital angular momentum diffusion and fix the orbital pericentre $r_{\mathrm{p}}$ and period $P$ throughout the evolution.

We show the radius evolution of the star as a function of time, for $m_{\mathrm{b}}=2$ and different pericentre distances, in Fig. 4. Similar experiments are done for other stellar masses and pericentre distances, and in this way, we find the critical pericentre distance $r_{\mathrm{p}, \mathrm{c}}$ below which cumulative tidal heating leads to expansion and disruption within $5 \mathrm{Myr}$ after the tidal capture. The critical pericentre $r_{\mathrm{p}, \mathrm{c}}$ as a function of $m_{\mathrm{b}}$ is shown in Fig. 3. We find $r_{\mathrm{p}, \mathrm{c}} \simeq 3$ au (roughly 
independent of the stellar mass) which corresponds to about $2 r_{\mathrm{T}, \mathrm{b}}$ instead of $4 r_{\mathrm{T}, \mathrm{b}}$ as suggested by Li \& Loeb (2013). The difference is because (1) the orbital periods in our cases are longer (as a result of higher eccentricity) allowing more cooling time, and (2) the stars between 1.5 and $6 \mathrm{M}_{\odot}$ have more centrally concentrated density profiles (than the 1 and $20 \mathrm{M}_{\odot}$ cases considered by Li \& Loeb 2013) and are less susceptible to tidal heating. In the next subsection, we study the angular momentum diffusion of the captured star in the $\mathrm{N}$ body environment near SgrA* under the (conservative) assumption that the star is disrupted if its pericentre is driven to be below $r_{\mathrm{p}, \mathrm{c}}$.

It should be noted that the age of the binary system is only loosely constrained to be between 30 and $100 \mathrm{Myr}(1 \sigma$ confidence interval). This uncertainty affects the radius and interior structure of the captured star especially if it is near the main-sequence turnoff. The main effect is that there is a maximum mass for the captured star $m_{\mathrm{b}, \max } \simeq 8,6$ (our fiducial case), $4.5 \mathrm{M}_{\odot}$ for the age of 30,50 (fiducial), $100 \mathrm{Myr}$, respectively. Doing similar experiments for an age of 30 or $100 \mathrm{Myr}$, we generally find $r_{\mathrm{p}, \mathrm{c}} \simeq 3$ au for $m_{\mathrm{b}}<m_{\mathrm{b} \text {, max }}$. As can be seen from Fig. 3 , the consequence of different $m_{\mathrm{b} \text {, max }}$ is that the captured star has lower mass and closer pericentre distance if we choose an older age. Since the survival probability is only weakly dependent on the mass of the bound star, the uncertainly due to binary age is subdominant compared to that from the relaxation time near SgrA $*$, which strongly affects the angular momentum diffusion of the post-capture orbit (see the next subsection).

\subsection{Angular momentum diffusion}

In this subsection, we consider the orbital evolution of the captured star in the $N$-body environment near SgrA $*$.

Unlike the quasi-circular orbit case where the scattering timescales in logarithmic orbital energy and angular momentum are comparable and equal to the relaxation time $t_{\text {rel }}$, here the newly captured star is in highly eccentric orbit with angular momentum $J \ll J_{\mathrm{c}}=\sqrt{G M / a_{\mathrm{b}}}$, so the time-scale for changing $\log J$ by unity, $t_{\mathrm{J}} \sim\left(J / J_{\mathrm{c}}\right)^{2} t_{\text {rel }}$, is much shorter. The quasi-steady state of a mass segregated cusp near SgrA $*$ can be described by a power-law density distribution $n_{*} \propto r^{-\alpha_{*}}$ with a mass-dependent slope that is steeper for heavier components (Bahcall \& Wolf 1977; Alexander \& Hopman 2009; Alexander 2017). The scattering rate crucially depends on the degree of mass segregation, especially the number of heavier stellar-mass $\mathrm{BHs}^{3}$ within 10 milliparsec $(\mathrm{mpc})$ from $\mathrm{SgrA} *$, since the relaxation rate is proportional to $\left\langle m^{2}\right\rangle=\int \mathrm{d} m m^{2}(\mathrm{~d} N / \mathrm{d} m)$ (Binney \& Tremaine 1987).

We carry out MC scattering ${ }^{4}$ simulations to track 5 Myr of angular momentum diffusion for the cases that survive direct tidal heating with $r_{\mathrm{p}}>r_{\mathrm{p}, \mathrm{c}}$. This is done using a simplified version of MC method by Shapiro \& Marchant (1978), see also Spitzer \& Shapiro (1972), Lightman \& Shapiro (1977), Hils \& Bender (1995), Hopman \& Alexander (2005), and Bar-Or \& Alexander

\footnotetext{
${ }^{3}$ Many works found that there may be $10^{2}$ to $10^{3}$ stellar-mass BHs within $10 \mathrm{mpc}$ of the GC (e.g. Freitag, Amaro-Seoane \& Kalogera 2006; Alexander \& Hopman 2009; Preto \& Amaro-Seoane 2010; Aharon \& Perets 2016; Generozov et al. 2018).

${ }^{4}$ Resonant relaxation (RR; Rauch \& Tremaine 1996) plays an important role for stars with much larger pericentre distances (e.g. the S-stars) but is strongly suppressed by rapid GR apsidal precession for the highly eccentric orbits considered here (Hopman \& Alexander 2006; Madigan, Hopman \& Levin 2011; Bar-Or \& Alexander 2016). RR is absent in our MC scattering experiment because the angular momentum change in each time step is due to uncorrelated 2-body interactions.
}

(2016). For each orbit, the angular momentum is perturbed by a drift term $\Delta_{1} J=J_{\mathrm{c}}^{2} P /\left(2 t_{\mathrm{rel}} J\right)$ and a stochastic term $\Delta_{2} J=$ $\pm\left(P / t_{\text {rel }}\right)^{1 / 2} J_{\mathrm{c}}$ (random choice between + and - ), where $P$ is the orbital period and $t_{\mathrm{rel}}(a)$ is the relaxation time at a distance of the SMA $a$ from the BH. This is because in the $J / J_{\mathrm{c}} \ll 1$ limit, the orbit undergoes random walk in $2 \mathrm{D} \mathrm{J}$-space with step length $\sqrt{\Delta J^{2}}$, and geometrically, one can show that the orbit-averaged mean change or drift in angular momentum is related to the mean squared change by $\Delta J=\Delta J^{2} / 2 J$ (Lightman \& Shapiro 1977$)$. Since $\left(P / t_{\text {rel }}\right)^{1 / 2} J_{\mathrm{c}} / J \ll$ 1 , the stochastic term $\Delta_{2} J$ is much larger than the drift term $\Delta_{1} J$, but both are important for time-scales of the order of $\left(J / J_{\mathrm{c}}\right)^{2} t_{\text {rel }}$ or longer.

The GW inspiral time for $r_{\mathrm{p}} \gtrsim 3$ au is longer than $100 \mathrm{Myr}$ and hence we ignore the angular momentum and energy carried away by GW. We also ignore energy perturbation by scattering because the (energy) relaxation time is much longer than $5 \mathrm{Myr}$ for all cases considered here, so the orbital energy and SMA stay fixed. For $J / J_{\mathrm{c}}$ $\ll 1$, dynamical friction only contributes to change in energy on a time-scale $\left(\langle m\rangle / m_{\mathrm{b}}\right) t_{\text {rel }} \sim t_{\text {rel }}$ and is hence ignored.

For a density distribution $n_{*} \propto r^{-\alpha_{*}}$, we obtain the relaxation time profile $t_{\text {rel }}(r) \propto r^{\alpha_{*}-3 / 2} /\left\langle m_{*}^{2}\right\rangle$, which increases with radius (since mass segregation gives $\alpha_{*}>3 / 2$ and $\left\langle m_{*}^{2}\right\rangle$ generally increases towards the supermassive $\mathrm{BH})$. We consider the following parametrized power-law

$t_{\mathrm{rel}}=t_{\mathrm{rel}, 0}\left(r / r_{0}\right)^{\alpha_{\mathrm{t}}}$,

normalized at $r_{0}=10 \mathrm{mpc}$ (near the initial SMA of the captured star) and we take $\alpha_{\mathrm{t}}=0.3$ roughly given by the Bahcall \& Wolf (1977, BW) profile for the heaviest objects $\alpha_{*}=7 / 4$ (appropriate for the innermost cusp where heavy BHs dominate relaxation). Since the SMA in our MC sample in Fig. 3 only spans a factor of a few (see equation 16), our results only depend weakly on the power-law index $\alpha_{\mathrm{t}}$. However, the normalization $t_{\mathrm{rel}, 0}$ is highly uncertain. In the case of the classical BW weak segregation (when stellar-mass BHs are relatively common), $t_{\mathrm{rel}, 0}$ is a few to $10 \mathrm{Gyr}$. On the other hand, Alexander \& Hopman (2009) show that, if the nuclear star cluster contains only a small fraction of stellar-mass BHs such that relaxation is dominated by lighter objects, then these BHs sink to the centre (by dynamical friction) and form a much steeper cusp. In this strongly segregated solution, the relaxation rate within 10's of mpc from SgrA $*$ may be enhanced by one or two orders of magnitude due to larger density of stellar-mass BHs. Above considerations motivate us to consider three different cases of $t_{\text {rel }, 0}=10^{10}, 10^{9}, 10^{8} \mathrm{yr}$. The shortest $t_{\text {rel, } 0}$ roughly corresponds to the strongly segregated model obtained by Aharon \& Perets (2016, right-hand panel of their fig. 1), which includes two populations of 10 and $30 \mathrm{M}_{\odot} \mathrm{BHs}$ (and the latter dominates relaxation).

For each IC (drawn from Fig. 3), we evolve the orbit for $5 \mathrm{Myr}$ during which the star is assumed to be rapidly disrupted if the pericentre distance falls below $r_{\mathrm{p}, \mathrm{c}}$. The simulation results are shown in Fig. 5. As expected, a large fraction of the cases are scattered to higher eccentricities and then disrupted. At the same time, some are scattered to lower eccentricities and survive. For both the empty and full loss-cone cases, the pericentre distributions of the surviving stars are similar.

The final survival probabilities for all cases are summarized in Table 1 . We find that the final survival probability of the captured star is between 5 per cent (for full loss cone and fastest relaxation) and 50 per cent (for empty loss cone and slowest relaxation). Of the surviving cases, $\gtrsim 90$ per cent are more massive than $2 \mathrm{M}_{\odot}$, which is mainly due to ICs rather than the slight preference for higher-mass stars to survive. 

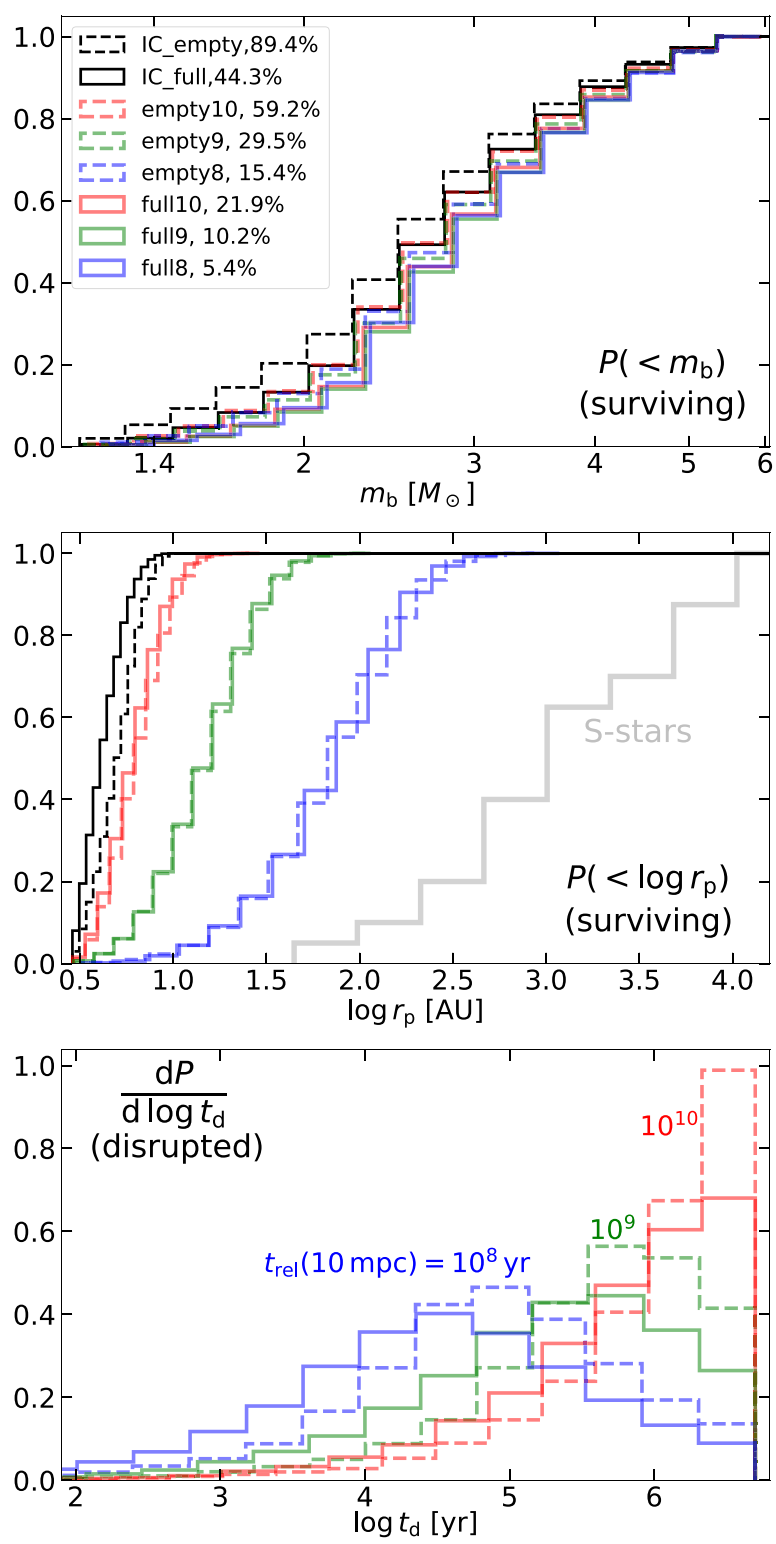

Figure 5. The mass (upper) and pericentre (middle panel) distributions for the cases that survive the tidal interactions after $5 \mathrm{Myr}$ of orbital evolution. The ICs, drawn from Fig. 3, are shown by black solid and dashed lines for the full and empty loss-cone cases, respectively. The red, green, blue histograms are for the three cases of relaxation time-scales $t_{\text {rel }}(10 \mathrm{mpc})=10^{10}, 10^{9}, 10^{8}$ $\mathrm{yr}$ (see equation 32), respectively. The final surviving percentage in each case is marked in the upper panel's legend. For shorter relaxation time, angular momentum diffusion is faster, more stars are tidally disrupted, and the pericentre distribution of the surviving cases is broader and centred at larger values. The surviving cases typically have pericentres smaller than most of the known S-stars as listed by Gillessen et al. (2017), which are shown by the silver line. In the mass distribution of the surviving cases, there is a weak preference for massive ones, mainly because massive stars typically have larger initial pericentres that are farther above $r_{\mathrm{p}, \mathrm{c}}$. The bottom panel shows the disruption time $t_{\mathrm{d}}$ at which the pericentre falls below $r_{\mathrm{p}, \mathrm{c}}$ (and we assume the star is instantaneously disrupted). All histograms are normalized as probability density (total area below each curve is unity).

In Table 2, we compared the expected properties of the surviving star to the three known S-stars (S2, S62, and S175) with the closest pericentres $\lesssim 100 \mathrm{au}$, which are seemingly consistent with our prediction for $t_{\text {rel }}(10 \mathrm{mpc}) \ll 1$ Gyr. The Hills mechanism predicts that the mass and ejection speed of the ejected star are related to the mass and period of the bound star by equation (17), $m_{\mathrm{e}} v_{\mathrm{e}, 1800}^{2} \simeq 1.5 m_{\mathrm{b}}(P / 10 \mathrm{yr})^{-2 / 3}$. According to this relation, we find that none of the three stars was associated with S5-HVS1: S2 is too massive/young, the estimated mass of S62 is too large for its SMA, and the estimated mass of S175 is too large for its SMA. However, S62 could be associated with a different HVS with $m_{\mathrm{e}} v_{\mathrm{e}, 1800}^{2} \simeq 7.5$ (slightly more massive and/or faster than S5-HVS1), and S175 could be associated with an HVS with $m_{\mathrm{e}} v_{\mathrm{e}, 1800}^{2} \simeq 1.2$ (slightly less massive and/or slower than S5-HVS1). The method developed in this work can generally applied to link future HVSs to S-stars and vice versa.

\section{IMPLICATIONS AND FUTURE OBSERVATION}

In this subsection, we discuss the implications on (1) the contribution to tidal disruption event (TDE) rate by binary break-ups, (2) the cumulative number of surviving captured stars within $10 \mathrm{mpc}$, and (3) using the relativistic orbits of the captured stars to measure the spin of SgrA $*$.

The Southern Stellar Stream Spectroscopy Survey $\left(S^{5} ; \mathrm{Li}\right.$ et al. 2019) had covered a small fraction of the sky $\Delta \Omega / 4 \pi \simeq$ $330 \mathrm{deg}^{2} / 4 \pi \ll 1$. There are potentially many more S5-HVS1-like stars in other areas of the sky and ejected earlier in time. The total ejection rate of HVSs in the mass range of 2.5 to $4 \mathrm{M}_{\odot}$ is estimated to be about $10^{-6} \mathrm{yr}^{-1}$ from recent observations (Brown, Geller \& Kenyon 2014; Brown et al. 2018). These stars typically have much lower ejection speeds $\sim 600 \mathrm{~km} \mathrm{~s}^{-1}$. Assuming a flat logarithmic binary separation distribution, Rossi, Kobayashi \& Sari (2014) predicted that $\gtrsim 20$ per cent (both full and empty loss cones) of HVSs should have speed around $1800 \mathrm{~km} \mathrm{~s}^{-1}$. Combining these two arguments gives about one S5-HVS1-like star every $5 \mathrm{Myr}$. This is in slight tension with the single detection by the $S^{5}$ survey, which only probed $\sim 1$ per cent of the spherical volume. This issue may be due to potentially anisotropic angular distribution of ejected stars, non-steady ejection rate, observational biases, and Poisson fluctuation.

In the following, we adopt a fiducial ejection rate of $\Gamma=$ $10^{-6} \Gamma_{-6} \mathrm{yr}^{-1}$ for S5-HVS1-like stars. Future observations, e.g. Gaia DR3 (Gaia Collaboration 2018) and Dark Energy Spectroscopic Instrument (DESI Collaboration 2016), will better measure this rate. Along with each ejection, there is a captured star. Very few cases are promptly disrupted. However, an order-unity fraction of the captured stars are disrupted after some delay as a result of cumulative tidal heating and angular momentum diffusion. Thus, the binary break-up channel provides a lower limit on the total TDE rate in the Milky Way to be $\gtrsim 5 \times 10^{-7} \Gamma_{-6} \mathrm{yr}^{-1}$ (taking the most conservative case of 50 per cent disruption). In these eccentric TDEs, the final full disruption is preceded by a few partial disruption events separated by about $20 \mathrm{yr}$ (similar to the white dwarf TDE cases considered by e.g. MacLeod et al. 2014; Vick, Lai \& Fuller 2017). The partial TDEs feed the BH at sub-Eddington rate and the accretion disc generates bright X-ray emission. Then, in the full disruption, about half of the star is fed to the BH (see Hayasaki et al. 2018, for a discussion of TDEs from bound stars), and both optical and X-ray emission may be generated near the Eddington luminosity (e.g. Strubbe \& Quataert 2009; Lodato \& Rossi 2011; Metzger \& Stone 2016; Dai et al. 2018; Lu \& Bonnerot 2020). Another possible but much less likely outcome is that, if the relaxation time is extremely long ( $>10 \mathrm{Gyr}$ ) such that angular momentum diffusion is slower than orbital circularization due to GW emission (such a situation may be realized for larger BH masses $\gtrsim 10^{7.5} \mathrm{M}_{\odot}$ ), then the captured star undergoes EMRI (see Metzger \& Stone 2017, for a discussion). 
Table 1. The probability corresponding to the different fate of the captured star for the empty and full loss-cone cases as shown in Fig. 3 . We consider three choices of relaxation time normalization $t_{\mathrm{rel}}(10 \mathrm{mpc})=10^{10}, 10^{9}, 10^{8} \mathrm{yr}$ (see equation 32), which depend on the (uncertain) outcome of mass segregation near SgrA $*$. The star (1) is promptly disrupted if $r_{\mathrm{p}}<0.5 r_{\mathrm{T}, \mathrm{b}}$ at binary break-up, (2) is disrupted after some delay (of less than $5 \mathrm{Myr}$ ) if $0.5 r_{\mathrm{T}, \mathrm{b}}<r_{\mathrm{p}}<r_{\mathrm{p}, \mathrm{c}}$, (3) survives until today if $r_{\mathrm{p}}>r_{\mathrm{p}, \mathrm{c}}$ holds throughout the angular momentum diffusion over the past 5 Myr. Generally, the final survival probability decreases for shorter relaxation time, because a larger fraction of cases are (randomly) scattered to lower angular momentum orbits and are disrupted.

\begin{tabular}{lcccr}
\hline Loss cone & $t_{\text {rel }}(10 \mathrm{mpc})[\mathrm{yr}]$ & $\begin{array}{c}\text { Prompt disruption (per cent) } \\
\left(r_{\mathrm{p}}<0.5 r_{\mathrm{T}, \mathrm{b}}\right)\end{array}$ & $\begin{array}{c}\text { Delayed disruption (per cent) } \\
\left(0.5 r_{\mathrm{T}, \mathrm{b}}<r_{\mathrm{p}}<r_{\mathrm{p}, \mathrm{c}}\right)\end{array}$ & $\begin{array}{c}\text { Survive (per cent) } \\
\left(r_{\mathrm{p}}>r_{\mathrm{p}, \mathrm{c}}\right)\end{array}$ \\
\hline empty & $10^{10}$ & 0 & 40.8 & 59.2 \\
& $10^{9}$ & 0 & 70.5 & 29.5 \\
full & $10^{8}$ & 0 & 84.6 & 15.4 \\
& $10^{10}$ & 0.6 & 77.5 & 21.9 \\
& $10^{9}$ & 0.6 & 89.2 & 10.2 \\
& $10^{8}$ & 0.6 & 94.0 & 5.4 \\
\hline
\end{tabular}

Table 2. Comparison between the properties of the captured companion of S5-HVS1 (if surviving) and the three known S-stars with pericentres closest to SgrA*: S62 (Peißker, Eckart \& Parsa 2020), S2 (GRAVITY Collaboration 2020), and S175 (also known as S0-104; Meyer et al. 2012; Gillessen et al. 2017). The SMA and period of the captured star are given by equations (16) and (17), respectively, and the pericentre has been modified by angular momentum diffusion over the past $5 \mathrm{Myr}$ (see Fig. 5). We find that none of the three stars are consistent with the captured star studied in this work: S2 is too massive/young, S62 has too small SMA for its estimated mass, and S175 has too large SMA for its estimated mass. However, modest variation in the parameters (mass, speed, and age) of the ejected star can be made to fit these three S-stars, which means that they are consistent with the Hills mechanism origin.

\begin{tabular}{lcccc}
\hline Properties & $\begin{array}{c}\text { Captured companion } \\
\text { of S5-HVS1 }\end{array}$ & S62 & S2 & S175 \\
\hline SMA (au) & $600-3000$ & 740 & 1030 & $3100-3800$ \\
$P(\mathrm{yr})$ & $8-90$ & 9.9 & 16 & $90-100$ \\
$r_{\mathrm{p}}(\mathrm{au})$ & $5-200$ & 18 & 120 & $35-50$ \\
$\mathrm{~K}-$ band magnitude & $19.5-15.5$ & $\sim 16$ & 14 & $\sim 7.5$ \\
mass $\left(\mathrm{M}_{\odot}\right)$ & $1.4-6$ & $\sim 5$ & $\gtrsim 10$ & $\sim 3.5$ \\
consistent with & - & too massive & too massive/young & $\begin{array}{c}\text { mass too small } \\
\text { for its SMA }\end{array}$ \\
\hline
\end{tabular}

The cumulative number of surviving bound stars in the past $5 \mathrm{Myr}$ is between $0.25 \Gamma_{-6}$ (for 5 per cent survival fraction) and $2.5 \Gamma_{-6}$ (for 50 per cent survival fraction). This is a lower limit because we do not take into account contributions from other lower mass binary systems and captures that occurred more than $5 \mathrm{Myr}$ ago (those systems may have a lower but non-zero surviving probability, depending on the relaxation time).

In Fig. 6, we show the estimated Keck NIRC2 (2nd generation near-infrared camera) $\mathrm{K}_{\mathrm{s}}$-band magnitude of a stellar population at different assumed ages of 30, 50, $100 \mathrm{Myr}$ for metallicity $[\mathrm{Fe} / \mathrm{H}]$ $=0.3$. The isochrones are generated by the public code SPISEA $^{5}$ (Hosek et al. 2020), using MESA Isochrones and Stellar Tracks (MIST) stellar evolution (Choi et al. 2016) and ATLAS atmosphere models (Castelli \& Kurucz 2003). We adopt extinction $A_{\mathrm{Ks}}=2.42$ mag as estimated by Fritz et al. (2011), see also Schödel et al. (2010). The majority ( $\gtrsim 90$ per cent) of the surviving cases should be brighter than the imaging limit $(18.5 \mathrm{mag})$ for the GRAVITY instrument at Very Large Telescope Interferometer (GRAVITY Collaboration 2020). We conclude that observations in the near future will likely find the captured companions of S5-HVS1-like stars.

If one of the captured stars considered in this work survived till today, it is most likely an undetected extreme member of the S-

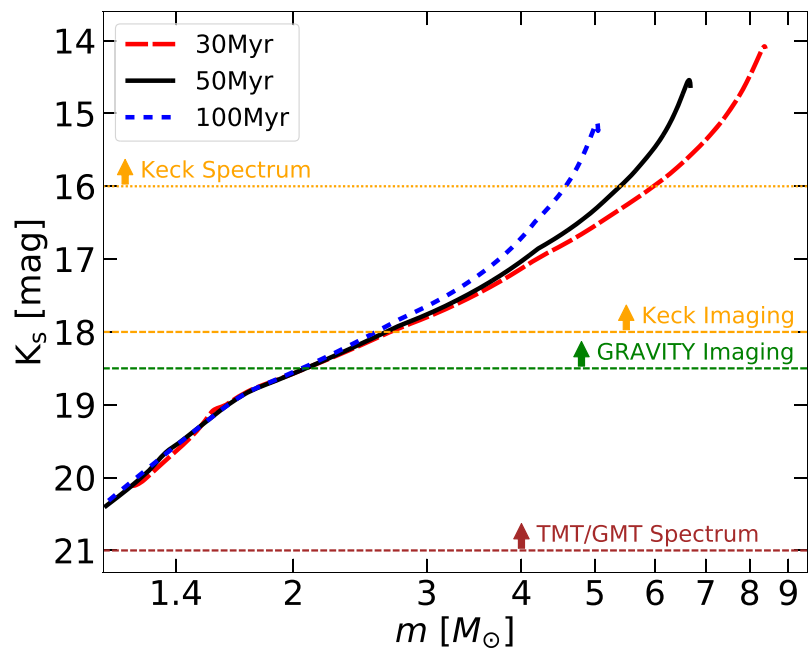

Figure 6. $\mathrm{K}_{\mathrm{s}}$-band magnitude for a stellar population at different ages of 30 , 50,100 Myr. We take metallicity $[\mathrm{Fe} / \mathrm{H}]=0.3$ (Koposov et al. 2020) and extinction $A_{\mathrm{Ks}}=2.42 \mathrm{mag}$ (Fritz et al. 2011). Detection limits for various instruments, as estimated by Do et al. (2017), are shown in horizontal lines including Keck, GRAVITY, and future Giant Magellan Telescope and Thirty Meter Telescope. 
star cluster with pericentre distance in the range 10 to $100 \mathrm{au}$. Its relativistic orbit provides an extraordinary probe of the spin of SgrA $*$. The Lense-Thirring (LT) precession per orbit is $\Phi_{\mathrm{LT}} \simeq$ $2.2 \times 10^{-4} \operatorname{rad}\left(r_{\mathrm{p}} / 30 \mathrm{au}\right)^{-3 / 2} \chi \sin i$, where $\chi$ is the dimensionless spin of the $\mathrm{BH}$ and $\sin i$ is the spin-orbit inclination. Taking a potential astrometric precision of $10 \mu \mathrm{as} \simeq 0.08 \mathrm{au}$ in projected distance, we see that the GRAVITY instrument is sensitive to BH spin provided that $\chi \sin i \gtrsim 0.3\left(r_{\mathrm{p}} / 30 \mathrm{au}\right)^{3 / 2}$ (see Waisberg et al. 2018, for a more detailed calculation).

\section{SUMMARY}

Due to its large ejection speed of $1800 \mathrm{~km} \mathrm{~s}^{-1}$, the hyper-velocity star S5-HVS1, was most likely produced by tidal break-up of a tight binary system by SgrA* (Hills 1988). We use general arguments to constrain the properties of the captured companion of S5-HVS1. The mass of the bound star is in the range $(1.2,6) \mathrm{M}_{\odot}$. It was initially in a highly eccentric orbit with typical pericentre distance 1-10 au and SMA $\sim 10^{3}$ au. We explore whether it survived until today by following its interior and orbital evolution for $5 \mathrm{Myr}$ after the capture.

For each pericentre passage, energy is injected into the star's normal oscillation modes via tidal excitation according to linear perturbation. We assume that the mode energy (mainly in the gmodes and sometimes f-modes) is quickly dissipated into heat in the radiative zone and then study the response of the star's interior structure by MESA simulations. We find that cumulative tidal heating leads to runaway radial expansion and disruption if the pericentre distance is below a critical value of about 3 au.

We then study the angular momentum diffusion in the dense stellar environment near SgrA* using a simple MC scattering simulation that captures the main physics. We find that the angular momentum of the captured star may evolve substantially from the initial value over the past $5 \mathrm{Myr}$, causing a large fraction of cases to be tidally disrupted and the pericentre distances of the surviving cases to be in the range 10 to $100 \mathrm{au}$. Overall, we find the surviving probability to be between 5 per cent and 50 per cent, depending on the local relaxation time of the captured orbit and whether the loss cone is empty or full where the binary originated.

The ejection rate of S5-HVS1-like stars, somewhere between $10^{-7}$ and $10^{-5} \mathrm{yr}^{-1}$, is highly uncertain due to observational incompleteness and Poisson error. For a median rate of $\Gamma=10^{-6} \mathrm{yr}^{-1}$, there are cumulatively between 0.25 and 2.5 surviving stars in similar orbits as the captured star studied in this work, which are potentially the most extreme members of the S-star cluster. They are detectable (typically $K_{\mathrm{s}} \lesssim 18.5 \mathrm{mag}$ ) by the GRAVITY instrument and by future Extremely Large Telescopes and will provide an extraordinary probe of the spin of SgrA $*$.

\section{ACKNOWLEDGEMENTS}

We thank Reinhard Genzel, Aleksey Generozov, Clément Bonnerot, Saul Teukolsky for useful discussions. WL was supported by the David and Ellen Lee Fellowship at Caltech. JF acknowledges support from an Innovator Grant from The Rose Hills Foundation, and the Sloan Foundation through grant FG2018-10515. HBP is grateful for the support from the Kingsley distinguished-visitor program at Caltech. TSL was supported by NASA through Hubble Fellowship grant HST-HF2-51439.001, awarded by the Space Telescope Science Institute, which is operated by the Association of Universities for Research in Astronomy, Inc., for NASA, under contract NAS526555. MWH acknowledges support by NASA through grant HSTGO-15199.001-A. TD was supported by NSF AAG AST-1909554.

\section{DATA AVAILABILITY}

The data underlying this article will be shared on reasonable request to the corresponding author.

\section{REFERENCES}

Aharon D., Perets H. B., 2016, ApJ, 830, L1

Alexander T., 2005, Phys. Rep., 419, 65

Alexander T., 2017, ARA\&A, 55, 17

Alexander T., Hopman C., 2009, ApJ, 697, 1861

Alexander T., Morris M., 2003, ApJ, 590, L25

Antonini F., Lombardi James C. J., Merritt D., 2011, ApJ, 731, 128

Bahcall J. N., Wolf R. A., 1977, ApJ, 216, 883

Bar-Or B., Alexander T., 2016, ApJ, 820, 129

Binney J., Tremaine S., 1987, Galactic Dynamics, Princeton University Press, Princeton, NJ

Bromley B. C., Kenyon S. J., Geller M. J., Barcikowski E., Brown W. R., Kurtz M. J., 2006, ApJ, 653, 1194

Brown W. R., Geller M. J., Kenyon S. J., Kurtz M. J., 2005, ApJ, 622, L33

Brown W. R., Geller M. J., Kenyon S. J., 2014, ApJ, 787, 89

Brown W. R., Lattanzi M. G., Kenyon S. J., Geller M. J., 2018, ApJ, 866, 39

Burkart J., Quataert E., Arras P., Weinberg N. N., 2012, MNRAS, 421, 983

Castelli F., Kurucz R. L., 2003, in Piskunov N., Weiss W. W., Gray D. F., eds, Proc. IAU Symp. 210, Modelling of Stellar Atmospheres. p. A20, preprint (arXiv:astro-ph/0405087)

Choi J., Dotter A., Conroy C., Cantiello M., Paxton B., Johnson B. D., 2016, ApJ, 823, 102

Cummings J. D., Kalirai J. S., Tremblay P. E., Ramirez-Ruiz E., Choi J., 2018, ApJ, 866, 21

Dai L., McKinney J. C., Roth N., Ramirez-Ruiz E., Miller M. C., 2018, ApJ, 859, L20

DESI Collaboration, 2016, preprint (arXiv:1611.00036)

Do T., Hees A., Dehghanfar A., Ghez A., Wright S., 2017, preprint (arXiv: 1711.06389)

Do T. et al., 2019, Science, 365, 664

Eggleton P. P., 1983, ApJ, 268, 368

Freitag M., Amaro-Seoane P., Kalogera V., 2006, ApJ, 649, 91

Fritz T. K. et al., 2011, ApJ, 737, 73

Fuller J., 2017, MNRAS, 472, 1538

Gaia Collaboration, 2018, A\&A, 616, A1

Generozov A., Madigan A.-M., 2020, ApJ, 896, 137

Generozov A., Stone N. C., Metzger B. D., Ostriker J. P., 2018, MNRAS, 478,4030

Genzel R., Eisenhauer F., Gillessen S., 2010, Rev. Mod. Phys., 82, 3121

Gillessen S. et al., 2017, ApJ, 837, 30

Gould A., Quillen A. C., 2003, ApJ, 592, 935

GRAVITY Collaboration, 2020, A\&A, 636, L5

Guillochon J., Ramirez-Ruiz E., 2013, ApJ, 767, 25

Hamers A. S., Perets H. B., 2017, ApJ, 846, 123

Hayasaki K., Zhong S., Li S., Berczik P., Spurzem R., 2018, ApJ, 855, 129

Hills J. G., 1988, Nature, 331, 687

Hils D., Bender P. L., 1995, ApJ, 445, L7

Hopman C., Alexander T., 2005, ApJ, 629, 362

Hopman C., Alexander T., 2006, ApJ, 645, 1152

Hosek Matthew W. J., Lu J. R., Lam C. Y., Gautam A. K., Lockhart K. E., Kim D., Jia S., 2020, AJ, 160, 143

Ivanov P. B., Papaloizou J. C. B., 2004, MNRAS, 347, 437

Koposov S. E. et al., 2020, MNRAS, 491, 2465

Kumar P., Goodman J., 1996, ApJ, 466, 946

Lee H. M., Ostriker J. P., 1986, ApJ, 310, 176

Li T. S. et al., 2019, MNRAS, 490, 3508

Li G., Loeb A., 2013, MNRAS, 429, 3040

Lightman A. P., Shapiro S. L., 1977, ApJ, 211, 244

Lodato G., Rossi E. M., 2011, MNRAS, 410, 359

Lu W., Bonnerot C., 2020, MNRAS, 492, 686

Lu J. R., Do T., Ghez A. M., Morris M. R., Yelda S., Matthews K., 2013, ApJ, 764, 155 
MacLeod M., Goldstein J., Ramirez-Ruiz E., Guillochon J., Samsing J., 2014, ApJ, 794, 9

McMillan S. L. W., McDermott P. N., Taam R. E., 1987, ApJ, 318, 261

Madigan A.-M., Levin Y., Hopman C., 2009, ApJ, 697, L44

Madigan A.-M., Hopman C., Levin Y., 2011, ApJ, 738, 99

Mainetti D., Lupi A., Campana S., Colpi M., Coughlin E. R., Guillochon J., Ramirez-Ruiz E., 2017, A\&A, 600, A124

Mardling R. A., 1995a, ApJ, 450, 722

Mardling R. A., 1995b, ApJ, 450, 732

Merritt D., 2013, Class. Quantum Gravity, 30, 244005

Merritt D., Alexander T., Mikkola S., Will C. M., 2011, Phys. Rev. D, 84, 044024

Metzger B. D., Stone N. C., 2016, MNRAS, 461, 948

Metzger B. D., Stone N. C., 2017, ApJ, 844, 75

Meyer L. et al., 2012, Science, 338, 84

Moe M., Di Stefano R., 2017, ApJS, 230, 15

Paxton B. et al., 2019, ApJS, 243, 10

Peißker F., Eckart A., Parsa M., 2020, ApJ, 889, 61

Perets H. B., 2009, ApJ, 690, 795

Perets H. B., Gualandris A., 2010, ApJ, 719, 220

Perets H. B., Šubr L., 2012, ApJ, 751, 133

Perets H. B., Hopman C., Alexander T., 2007, ApJ, 656, 709

Perets H. B., Gualandris A., Kupi G., Merritt D., Alexander T., 2009, ApJ, 702,884

Peters P. C., 1964, Phys. Rev., 136, 1224

Press W. H., Teukolsky S. A., 1977, ApJ, 213, 183

Preto M., Amaro-Seoane P., 2010, ApJ, 708, L42

Rauch K. P., Tremaine S., 1996, New Astron., 1, 149

Rossi E. M., Kobayashi S., Sari R., 2014, ApJ, 795, 125

Ryu T., Krolik J., Piran T., Noble S., 2020, ApJ, 904, 98

Sari R., Kobayashi S., Rossi E. M., 2010, ApJ, 708, 605

Schödel R., Najarro F., Muzic K., Eckart A., 2010, A\&A, 511, A18

Shapiro S. L., Marchant A. B., 1978, ApJ, 225, 603

Spitzer Lyman J., Shapiro S. L., 1972, ApJ, 173, 529

Stone N. C., Vasiliev E., Kesden M., Rossi E. M., Perets H. B., Amaro-Seoane P., 2020, Space Sci. Rev., 216, 35

Strubbe L. E., Quataert E., 2009, MNRAS, 400, 2070

Townsend R. H. D., Teitler S. A., 2013, MNRAS, 435, 3406

Vanbeveren D., De Donder E., Van Bever J., Van Rensbergen W., De Loore C., 1998, New Astron., 3, 443

Vick M., Lai D., Fuller J., 2017, MNRAS, 468, 2296

Vick M., Lai D., Anderson K. R., 2019, MNRAS, 484, 5645

Waisberg I. et al., 2018, MNRAS, 476, 3600

Weinberg N. N., Arras P., Quataert E., Burkart J., 2012, ApJ, 751, 136

Weissbein A., Sari R., 2017, MNRAS, 468, 1760

Wu Y., Goldreich P., 2001, ApJ, 546, 469

Yu Q., Tremaine S., 2003, ApJ, 599, 1129

Zhang F., Lu Y., Yu Q., 2013, ApJ, 768, 153

\section{APPENDIX A: TIDAL COUPLING CONSTANT}

Consider a star of mass $M_{*}$ and radius $R_{*}$ in a parabolic orbit with pericentre distance $r_{\mathrm{p}}=\chi R_{*}\left(M / M_{*}\right)^{1 / 3}$. The spatial coupling between a stellar oscillation mode $n l$ and the tidal potential is given by (Burkart et al. 2012; Fuller 2017)

$Q_{n l}=-(2 l+1) \delta \Phi_{n l}\left(R_{*}\right) /(4 \pi)$,

where $\delta \Phi_{n l}\left(R_{*}\right)$ is the Eulerian gravitational perturbation at the stellar surface in units of $G M_{*} / R_{*}$, and $\omega_{n}$ is the frequency of the eigenmode in units of $\left(R_{*}^{3} / G M_{*}\right)^{1 / 2}$. For each MESA stellar model, we compute

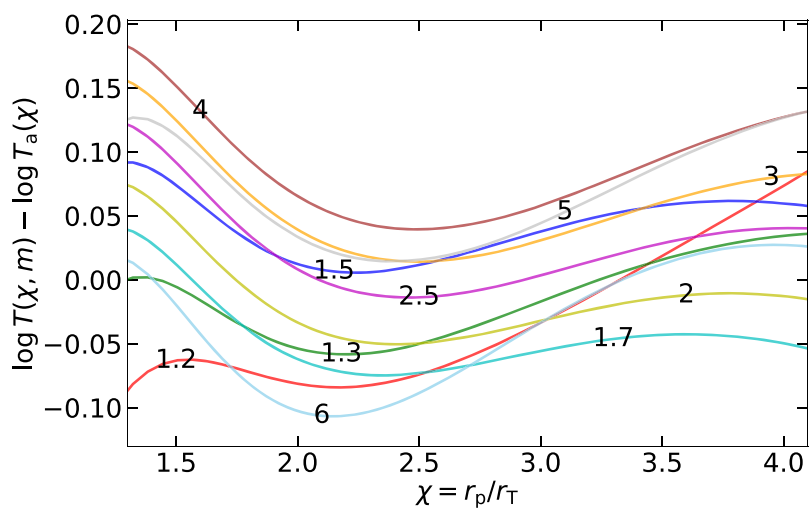

Figure A1. Tidal coupling coefficient $T\left(\chi, M_{*}\right)$ for different stellar masses (in $\mathrm{M}_{\odot}$ ) marked on each line. Our numerical MESA simulations are based on accurate $T\left(\chi, M_{*}\right)$ for each stellar mass. Here, for intuitive understanding, we show the residual after subtracting the simple analytical expression of $\log T_{\mathrm{a}}(\chi)$ in equation (A4), which provides a good description to within 15 per cent for $1.5<M_{*}<6 \mathrm{M}_{\odot}$ and $1.5<\chi<4$. The coupling coefficients for lower masses $M_{*}<1.5 \mathrm{M}_{\odot}$ are larger due to their more convective (and less centrally concentrated) structures, so we have added $\Delta \log T_{\mathrm{a}}=0.2$ (or 0.55 ) to equation (A4) for the case of $M_{*}=1.3 \mathrm{M}_{\odot}$ (or 1.2 $\mathrm{M}_{\odot}$ ). The stellar models all have main-sequence age of $50 \mathrm{Myr}$ and $Z=0.03$.

$\delta \Phi_{n l}\left(R_{*}\right)$ and hence $Q_{n l}$ with GYRE (Townsend \& Teitler 2013). The temporal coupling between the orbit and the stellar oscillation is given by

$K_{n l m}=\frac{W_{l m}}{2 \pi} 2^{3 / 2} \chi^{3 / 2} I_{l m}\left(\chi^{3 / 2} \omega_{n}\right)$,

where $\chi$ is the ratio between pericentre distance and tidal disruption radius, $W_{20}=-\sqrt{\pi / 5}, W_{2 \pm 2}=\sqrt{3 \pi / 10}$, and the function $I_{l m}(y)$ is given by equation (43) of Press \& Teukolsky (1977). Then the tidal coupling constant is given by

$T\left(\chi, M_{*}\right)=2 \pi^{2} \sum\left|Q_{n l}\right|^{2}\left|K_{n l m}\right|^{2}$.

Our MESA simulations including tidal heating are based on cubic polynomial fits to $\log [T(\chi)]$ for each stellar mass considered, which are accurate to within 2 per cent.

For intuitive understanding, we find that the tidal coupling constant can be roughly described by the following simple analytical expression

$\log T\left(\chi, M_{*}\right) \simeq-0.9(\chi+1)$

valid to within 15 per cent for $1.5<M_{*}<6 \mathrm{M}_{\odot}$ and $1.5<\chi<$ 4 , as shown in Fig. A1. Therefore, the dimensionless tidal energy deposition per pericentre passage is

$\Delta \widetilde{E}=\frac{\Delta E}{G M_{*}^{2} / R_{*}} \simeq \chi^{-6} 10^{-0.9(\chi+1)}$,

which means $\Delta \widetilde{E}(\chi=1.5) \approx 4.9 \times 10^{-4}, \Delta \widetilde{E}(2) \approx 3.1 \times 10^{-5}$, $\Delta \widetilde{E}(3) \approx 3.4 \times 10^{-7}$, and $\Delta \widetilde{E}(4) \approx 7.7 \times 10^{-9}$.

This paper has been typeset from a $\mathrm{T}_{\mathrm{E}} \mathrm{X} / \mathrm{LT} \mathrm{E} \mathrm{X}$ file prepared by the author. 